\title{
FUNDAMENTOS METATEÓRICOS DE LA CIENCIA EMPÍRICA DE LA LITERATURA
}

\author{
FRANCISCO CHICO RICO \\ (Universidad de Alicante)
}

1. La ciencia empírica de la literatura («Empirische Literaturwissenschaft») representa en la actualidad una concepción científico-literaria que pretende ser fundadamente revolucionaria dentro del ámbito general de los estudios teórico-literarios y crítico-literarios contemporáneos. Concebida y desarrollada fundamentalmente por el profesor Siegfried J. Schmidt ${ }^{1}$ a partir de 1974 en colaboración con el grupo de investigación NIKOL ${ }^{2}$, la ciencia empírica de la literatura intenta poner fin a la situación de heterogeneidad de concepciones ${ }^{3}$ y de parcialización teórico-me-

1 La más completa exposición de la concepción y el desarrollo de la ciencia empírica de la literatura se encuentra en S. J. Schmidt, Grundriss der Empirischen Literaturwissenschaft. Der gesellschaftliche Handlungsbereich Literatur, Braunschweig/Wiesbaden, Vieweg (Konzeption Empirische Literaturwissenschaft, 1, 1), 1980 y en S. J. Schmidt, Grundriss der Empirischen Literaturwissenschaft. Zur Rekonstruktion literaturwissenschaftlicher Fragestel/ungen in einer Empirischen Theorie der Literatur, Braunschweig/Wiesbaden, Vieweg (Konzeption Empirische Literaturwissenschaft, I, 2), 1982. Para un estudio introductorio y más general vid., entre otros, S. J. Schmidt, "Empirische Literaturwissenschaft as Perspective", en Poetics, 8, 1979, pp. 557-568; S. J. Schmidt, «Empirical Studies in Literature: Introductory Remarks», en Poetics, 10, 1981, pp. 317-336; S. J. Schmidt, «Die Empirische Literaturwissenschaft: Ein neues Paradigma», en SPIEL, 1, 1, 1982, pp. 5-25 y H. Hauptmeier - S. J. Schmidt, Einführung in die Empirische Literaturwissenschaft, Braunschweig/ Wiesbaden, Vieweg, 1985. Vid. también las explicaciones acerca de la ciencia empírica de la literatura realizadas en un marco más general por J. M. Pozuelo, La lengua literaria, Málaga, Ágora, 1983, pp. 78-80.

2 Peter Finke, Walther Kindt, Siegfried J. Schmidt, Jan Wirrer y Reinhold Zobel fueron los primeros integrantes de este grupo en el marco de la Universidad de Bielefeld. En 1980 el grupo fue ampliado con Achim Barsch, Heimut Hauptmeier, Dietrich Meutsch, Gebhard Rusch y Reinhold Viehoff, de la Universidad de Siegen, donde desde ese año se encuentra su director, Siegfried J. Schmidt. Desde 1984 la sección de Siegen del grupo NIKOL forma parte del Instituto para la Investigación Empírica de la Literatura y de los Medios de Comunicación («Institut für Empirische Literatur- und Medienforschung") o Instituto LUMIS ("Literatur- und Medienforschung in Siegen»), del que también es director el profesor Schmidt.

3 Peter Finke expone claramente esta situación cuando escribe: «In den meisten Fällen liegt eine Disziplin irgendwo dazwischen. Der Maßstab, mit dem hier gemessen wird, ist der einer relativen Homogeneität oder Inhomogeneität, bezogen auf den Grad, zu dem eine Wissenschaftlergemeinschaft gemeinsame 
todológica 4 que ha caracterizado a esta disciplina filológica desde hace mucho tiempo para poder hacer de ella una ciencia sólida desde un punto de vista teórico y relevante desde un punto de vista práctico ${ }^{5}$, es decir, para poder convertirla en lo que para Thomas $\mathrm{S}$. Kuhn es una ciencia normal ${ }^{6}$.

1.1. Desde esta perspectiva puede decirse, utilizando también términos kuhnianos, que la ciencia literaria en su estado actual, así como la ciencia lingüística ${ }^{7}$ y otras muchas pertenecientes al ámbito de las llamadas ciencias humanas, puesto que son pluriconcepcionales ${ }^{8} \mathrm{e}$ inestables en relación con sus fundamentos teórico-científicos más elementales, se encuentran, al menos en parte, en fases preparadigmáticas de su desarrollo. Por el contrario, aquellas disciplinas que, como la física, la química y otras muchas consideradas como ciencias naturales, son concepcionalmente homogéneas atraviesan desde hace mucho tiempo, al menos en parte, fases paradigmáticas ${ }^{9}$. En efecto, mientras que estas últimas han sido elaboradas y desarrolladas a partir de la existencia de un paradigma ${ }^{10}$, las primeras no

Überzeugungen teilt. Dieser Homogeneitätsgrad ist dann besonders gering, wenn die Überzeugungsdifferenzen den Bereich treffen, der zumeist vage als die Basis oder der Bereich der Grundlagen der betreffenden Disziplin bezeichnet wird. Die Literaturwissenschaft in ihrem gegenwärtigen Entwicklungszustand gilt allgemein als ein prägnantes Beispiel." (P. Finke, Konstruktiver Funktionalismus. Die wissenschaftstheoretische Basis einer empirischen Theorie der Literatur, Braunschweig/Wiesbaden, Vieweg (Konzeption Empirische Literaturwissenschaft, II), 1982, p. 2).

4 En nuestro país ha sido principalmente Antonio García Berrio quien ha denunciado enérgicamente esta situación incidiendo sobre todo en la consideración restringida del objeto de estudio por parte de las diferentes tendencias, cada vez más excluyentes, de la actual ciencia literaria (cfr. A. García Berrio, Significado actual del formalismo ruso. La doctrina de la escuela del método formal ante la poética y la lingüistica modernas, Barcelona, Planeta, 1973, pp. 90-91; A. García Berrio, "Crítica formal y función crítica», en Lexis, 1, 2, 1977, pp. 187-209; A. García Berrio, "Texto y oración. Perspectivas de la lingüística textual», en J. S. Petöfi - A. García Berrio, Lingüística del texto y critica literaria, Madrid, Comunicación, 1978, pp. 243-264; A. Garcia Berrio, «Lingüística, literaridad/poeticidad. (Gramática, Pragmática, Texto)», en 1616. Anuario de la Sociedad Española de Literatura General y Comparada, 2, 1979, pp. 125-170; A. García Berrio, "Más altá de los 'ismos': Sobre la imprescindible globalidad crítica», en P. Aullón de Haro (coord.), Introducción a la crítica literaria actual, Madrid, Playor, 1984, pp. 347-387 y A. García Berrio, "Retórica como ciencia de la expresividad. (Presupuestos para una Retórica General)", en Estudios de Lingüistica, 2, 1984, pp. 7-59). En este sentido Tomás Albaladejo ha defendido, desde un punto de vista semiótico-textual, la absoluta necesidad de estudiar la obra literaria en el seno del hecho literario, es decir, el complejo acontecimiento en el que la obra de arte verbal es comunicada por un productor a un receptor en una situación contextual determinada y en relación con el mundo o mundos que configuran el sistema general de referencias del que participan efectivamente todos y cada uno de estos elementos (cfr., muy especialmente, $T$. Albaladejo, "Componente pragmático, componente de representación y modelo lingüistico-textual", en Lingua e Stile, XVIII, 1, 1983, pp. 3-46; T. Albaladejo, "La crítica lingüística", en P. Aullón de Haro (coord.), Introducción a la critica literaria actual, cit., pp. 141-207, 186 ss. y T. Albaladejo, Teoría de los mundos posibles y macroestructura narrativa. Análisis de las novelas cortas de Clarín, Alicante, Universidad de Alicante, 1986).

5 Cfr. P. Finke, Konstruktiver Funktionalismus, cit., pp. 38-42.

6 Cfr. Th. S. Kuhn, La estructura de las revoluciones cientificas, Madrid, Fondo de Cultura Económica, 1975 , pp. 33 ss.

7 En relación con la ciencia lingüistica vid., muy especialmente, P. Finke, Grundlagen einer linguistischen Theorie. Empirie und Begründung in der Sprachwissenschaft, Braunschweig/Wiesbaden, Vieweg, 1979.

8 Cfr. P. Finke, Konstruktiver Funktionalismus, cit., pp. 1-8, 25-26, 43-46.

9 Cfr. Ibid., pp. 53-55.

10 Para Thomas S. Kuhn, según una de sus últimas definiciones, «Ein Paradigma ist das, was den Mitgliedern einer wissenschaftlichen Gemeinschaft, und nur ihnen, gemeinsam ist." (Th. S. Kuhn, Die Entstehung des Neuen. Studien zur Struktur der Wissenschaftsgeschichte, Frankfurt/M., Suhrkamp, 1977, 
han conseguido todavía el establecimiento de uno de estos sistemas generales de condiciones teórico-científicas fundamentales que se encargan de regular todos los procesos de investigación en cada uno de sus ámbitos.

1.1.1. Para los miembros del grupo NIKOL la creación de un sistema general de condiciones científico-literarias fundamentales válido para la elaboración y el desarrollo racionales de una moderna ciencia de la literatura, considerada como ciencia normal, no puede entenderse, ante todo, sin una actividad científica orientada hacia la construcción de teorias, pues éstas, intuitivamente hablando, son el resultado del trabajo de grupos científicos. En ningún caso se trata de productos dados por la naturaleza, sino de constructos ${ }^{11}$ que constituyen instrumentos para la solución, consecuentemente siempre aproximativa, de los problemas que se presentan en una determinada sección de la realidad ${ }^{12}$.

1.1.1.1. Dicha actividad científica constructiva es llevada a cabo, en el ambito concreto de la ciencia empírica de la literatura, con el apoyo de una concepción científico-literaria no conservadora («nicht-konservative Literaturwissenschaftskonzeption" $)^{13}$, ya que la conservación de determinadas condiciones teórico-científicas fundamentales en diferentes sistemas generales, según los representantes de esta propuesta, puede influir negativamente sobre la capacidad creadora de los científicos. De acuerdo con los postulados teórico-cognitivos del constructivismo radical («radikaler Konstruktivismus»), desarrollado desde diferentes puntos de vista especialmente por los biólogos Humberto R. Maturana y Francisco J. Varela, por el cibernético Heinz von Foerster y por el psicólogo Ernst von Glasersfeld ${ }^{14}$, el grupo

p. 390). Vid. también, en relación con este concepto, Th. S. Kuhn, La estructura de las revoluciones cientificas, cit. Peter Finke, por su parte, para referirse a este imprescindible elemento teórico-científico de base habla, como también lo hacemos nosotros, de concepciones: «Konzeptionen —dice— sind [...] eine Teilklasse der möglichen Metatheorien einer Theorie, nämlich genau diejenige Teilklasse, die die möglichen und faktischen Systeme von Rahmenbedingungen, unter denen die objekttheoretische Forschung in einer Disziplin möglich wird, definieren.» (P. Finke, Konstruktiver Funktionalismus, cit., p. 26).

11 A propósito del concepto de "constructo» vid. S. K. Šaumjan, "Concerning the Logical Basis of Linguistic Theory", en Proceedings of the Ninth International Congress of Linguists, Cambridge, Mass., August 27-31, 1962, The Hague, Mouton, 1964, p. 155; L. Heilmann - E. Rigotti, «ll generativismo sintattico e semantico. Introduzione", en L. Heilmann - E. Rigotti (eds.), La linguistica: aspetti e problemi, Bologna, II Mulino, 1975, p. 208 y T. Albaladejo, "Considerazioni sulla teoria linguistica testuale», en Studi italiani di linguistica teorica ed applicata, 7, 3, 1978, pp. 363-365.

12 Cfr. P. Finke, Konstruktiver Funktionalismus, cit., pp. 22-25.

13 La denominación del grupo de investigación NIKOL está formada precisamente por las iniciales de los elementos léxicos del sintagma alemán «nicht-konservative Literaturwissenschaftskonzeption».

14 Con relación a esta importantísima teoria cognitiva vid., fundamentalmente, H. R. Maturana, "Biology of Cognition", en H. R. Maturana - F. J. Varela, Autopoiesis and Cognition. The Realization of the Living, Dordrecht: Holiand, D. Reidel Publishing Company, 1980, pp. 1-58; H. R. Maturana, Erkennen: Die Organisation und Verkörperung von Wirklichkeit. Ausgewählte Arbeiten zur biologischen Epistemologie, Braunschweig/Wiesbaden, Vieweg, 1985; H. Von Foerster, «Thoughts and Notes on Cognition", en P. L. Garvin (ed.), Cognition: A Multiple View, New York, Spartan Books, 1970, pp. 25-48; H. von Foerster, "Kybernetik einer Erkenntnistheorie", en W. D. Keidel - W. Händler - W. Spreng (Hrsg.), Kybernetik und Bionik, München/Wien, Oldenbourg, 1974, pp. 27-46; H. von Foerster, «Das Konstruieren einer Wirklichkeit», en P. Watzlawick (Hrsg.), Die erfundene Wirklichkeit. Wie wissen wir, was wir zu wissen glauben? Beiträge zum Konstruktivismus, München, Piper, 1985, pp. 39-60; E. von Glasersfeld, "Signs, Communication and Language", en Journal of Human Evolution, 3, 1974, pp. 465-474; E. von Glasersfeld, "On the Concept of Interpretation», en Poetics, 12, 1983, pp. 207-218; E. von Glasersfeld, "Einführung in den radikalen Konstruktivismus», en $P$. 
de investigación NIKOL considera conservadoras las condiciones científico-literarias de la textualidad, entendida como característica esencial del concepto de «literatura» ${ }^{15}$, y de la interpretación, en tanto que problema central de la investigación científico-literaria tradicional ${ }^{16}$. Los miembros de este grupo van a calificar, por ello, de conservadora toda concepción científico-literaria que contenga una de estas dos condiciones ${ }^{17}$. Frente a ellas, serán las consideraciones de la literatura como un complejo sistema social de acciones ${ }^{18}$ y de la investigación empírica de ese sistema como la función más importante de la ciencia empírica de la literatura ${ }^{19}$ dos de los presupuestos científico-literarios fundamentales que van a permitirles calificar la concepción de esta ciencia de no conservadora ${ }^{20}$.

1.2. La teoria de la ciencia o metateoria que permite tanto la construcción de teorías como el establecimiento de condiciones teórico-científicas fundamentales no conservadoras en las diferentes concepciones elaboradas según sus presupuestos es, para el grupo NIKOL, el llamado funcionalismo constructivo («konstruktiver Funktionalismus») ${ }^{21}$. EI funcionalismo constructivo es una teoría de la ciencia empírica y revisable, es decir, una teoría interpretable a partir de la observación de la realidad y perfeccionable a través de la experiencia, que describe un posible proceso de creación de teorías también empíricas y revisables en el amplio marco de las ciencias preparadigmáticas. Por ello puede entenderse como un generador abstracto de teorías empíricas ${ }^{22}$. Esta teoría de la ciencia va a constituir,

\begin{abstract}
Watzlawick (Hrsg.), Die erfundene Wirklichkeit, cit., pp. 16-38; S. J. Schmidt, "Empirische Literaturwissenschaft as Perspective», cit.; S. J. Schmidt, "Fictionality in Literary and Non-Literary Discourse», en Poetics, 9 , 1980, pp. 525-546; S. J. Schmidt, «Unsere Welt - und das ist ailes", en Merkur. Deutsche Zeitschrift für europäisches Denken, 36, H. 403-414, 1982, pp. 356-366; S. J. Schmidt, «Text, Subjekt und Gesellschaft. Aspekte einer konstruktivistischen Semantik», en M. Faust et al. (Hrsg.), Allgemeine Sprachwissenschaft, Sprachtypologie und Textinguistik. Festschrift für P. Hartmann, Tübingen, Narr, 1983, pp. 55-71; S. J. Schmidt, "The Fiction is that Reality exists. A Constructivist Model of Reality, Fiction, and Literature», en Poetics Today, 5, 2, 1984, pp. 253-274; S. J. Schmidt, “Comprender textos - Interpretar textos», en Estudios de Lingüistica, 4, 1987, pp. 9-32; H. Hauptmeier - R. Viehoff, "Empirical Research on the Basis of Bio-Epistemology. A New Paradigm for the Study of Literature?», en Poetics Today, 4, 1, 1983, pp. 153-171 y $\mathrm{H}$. Hauptmeier - S. J. Schmidt, Einführung in die Empirische Literatur wissenschaft, cit., pp. 26-29.

15 "Es ist wohl nicht übertrieben -escribe Finke-, wenn ich behaupte, daß nahezu a // e Konzeptionen der Literatur wissenschaft, die vorgelegt worden sind, über einem konservativen Literaturbegriff errichtet und damit konservative Konzeptionen sind: die bekannte werkimmanente Konzeption (Staiger, Kayser, Alewyn u.a.) kann hier als Paradigma gelten. Aber auch fast alle anderen etablierten Konzeptionen sind konservativ: strukturalistische Konzeptionen (Barthes, Goldmann, Greimas, Jakobson, Kristeva, Lotman, Todorov, Tel Quel), die Konzeption des new criticism, die Konzeptionen von Jauss, Iser, Conrady u.a., die semiotische Konzeption Wienolds, die textwissenschaftlich/linguistischen Konzeptionen Ihwes und Schmidts, die literatursoziologischen Konzeptionen Adornos, Benjamins, aber auch z.B. Groebens empirische Rezeptionstheorie.» (P. Finke, Konstruktiver Funktionalis mus, cit., p. 28).
\end{abstract}

16 Cfr. Ibíd., p. 29.

17 Cfr. Ibid., pp. 26-30.

18 Cfr. Ibíd., p. 31.

19 Cfr. Ibíd., p. 29.

20 Cfr. Ibíd., pp. 30-34.

21 Cfr. P. Finke, «A Sketch of Constructive Functionalism», en Poetics, 10, 1981, pp. 337-355 y P. Finke, Konstruktiver Funktionalismus, cit.

22 Cfr. P. Finke, "A Sketch of Constructive Functionalism", cit., pp. 344 ss. y P. Finke, Konstruktiver Funktionalismus, cit., pp. 185-201, 220-230. 
por lo tanto, la teoria que describa y explique la estructura y la función de la ciencia empírica de la literatura, teniendo en cuenta que en el ámbito del funcionalismo constructivo no es la estructura la que determina la función de una teoria, sino al contrario. De la razón práctica (función) de cualquier teoría construida a partir de los presupuestos del funcionalismo constructivo dependerá su razón teórica (estructura) ${ }^{23}$. El funcionalismo constructivo, invirtiendo de este modo el orden normal de las determinaciones teórico-cientificas, pretende convertir nuestro conocimiento científico estructural en un conocimiento funcional mucho más comprehensivo ${ }^{24}$.

1.2.1. Esta teoria ha sido, como la ciencia empírica de la literatura, desarrollada interdisciplinariamente en el seno del grupo NIKOL, siendo en este caso su principal responsable Peter Finke ${ }^{25}$. El profesor Finke, para ello, ha partido de una versión modificada de la teoría analítica de la ciencia desarrollada en detalle y demostrada en teorias de la física matemática por Joseph $D$. Sneed ${ }^{26}$. Se trata de la visión no proposicional («non-statement view») o concepción conceptual («Begriffskonzeption") de las teorías ${ }^{27}$.

1.2.1.1. Para esta concepción científica una teoría empírica es considerada como el significado de un predicado. Por un predicado se entiende, en el marco de la lógica moderna, una función proposicional del tipo « $F(x) » 0$, utilizando una expresión lingüística, « $x$ es $F$ » Puesto que el significado de uno de estos predicados

23 Cfr. P. Finke, Konstruktiver Funktionalismus, cit., pp. 71 ss.

24 Cfr. P. Finke, «A Sketch of Constructive Functionalism», cit., pp. 339-343 y P. Finke, Konstruktiver Funktionalismus, cit., pp. 43 ss, donde el autor lieva a cabo una crítica razonada del estructuralismo reconstructivo frente a las propuestas del funcionalismo constructivo.

25 Konstruktiver Funktionalismus. Die wissenschaftstheoretische Basis einer empirischen Theorie der Literatur es la primera presentación sistemática de la concepción de esta teoria de la ciencia, elaborada con la finalidad fundamental de llevar a cabo la fase de construcción de una base teórica más satisfactoria para el estudio de la literatura. A propósito de este tema vid. también P. Finke. "A Sketch of Constructive Functionalism", cit.

26 Cfr. J. D. Sneed, The Logical Structure of Mathematical Physics, Dordrecht:Holland, D. Reidel Publishing Company, 1971 y J. D. Sneed, «Philosophical Problems in the Empirical Science of Science: A Formal Approach", en Erkenntnis, 10, 1976, pp. 115-146.

27 Las ideas de esta visión o concepción de las teorias se remontan a E. W. Adams (cfr. E. W. Adams, "The Foundations of Rigid Body Mechanics and the Derivation of its Laws from those of Particle Mechanics", en J. Henkin - P. Suppes - A. Tarski (ed.), The Axiomatic Method, Amsterdam, North-Holland Publishing Company, 1959, pp. 250-265) y, además de haber sido aplicadas por Finke al ámbito de la ciencia de la literatura, también han servido para reconstruir teorías psicológicas y económicas (cfr., por ejemplo, W. Balzer, Empirische Theorien: Modelle - Strukturen - Beispiele, Braunschweig/Wiesbaden, Vieweg. 1982 y W. Diederich, Strukturalistische Rekonstruktionen, Braunschweig/Wiesbaden, Vieweg, 1981). No entramos aquí en una labor de explicación pormenorizada de esta concepción. Para ello vid., fundamentalmente, W. Diederich (Hrsg.), Theorien der Wissenschaftsgeschichte. Beiträge zur diachronen Wissenschaftstheorie, Frankfurt/M., Suhrkamp, 1974; C. U. Moulines, "Approximate Application of Empirical Theories: A General Explication», en Erkenntnis, 10, 1976, pp. 201-227; W. Stegmülier, Probleme und Resultate der Wissenschaftstheorie und Analytischen Philosophie. Band II: Theorie und Erfahrung. Zweiter Halbband: Theorienstrukturen und Theoriendynamik, Berlin/Heidelberg/New York, Springer-Verlag, 1973 (publicado en español como Estructura y dinámica de teorias, Barcelona, Ariel, 1983); W. Stegmüller, The Structuralist View of Theories, Berlin/Heidelberg/New York, Springer-Verlag, 1979; W. Stegmüller, Neue Wege der Wissenschaftsphilosophie, Berlin/Heidelberg/New York, Springer-Verlag, 1980; P. Finke, Grundlagen einer linguistischen Theorie, cit.; P. Finke, Konstruktiver Funktionalismus, cit. y H. Hauptmeier - S. J. Schmidt, Einführung in die Empirische Literaturwissenschaft, cit. 
es un concepto, una teoría empírica de este tipo puede ser considerada, del mismo modo, como un concepto. A partir de aquí se puede asignar a predicados concretos como « $x$ es una acción literaria de producción» 0 « $x$ es una novela» una proposición especificadora de los objetos de la realidad que pueden estar comprendidos dentro del concepto denotado por dicho predicado, es decir, podemos asignar $a x$ nombres de objetos reales. Con ello una teoría empírica, según la visión no proposicional o concepción conceptual de las teorías que comentamos, está compuesta por una ley fundamental, que es el significado de un predicado, aplicable a determinadas secciones de la realidad. La formulación de esta ley constituye el requisito estructural imprescindible y central de las teorías empíricas. Una teoría empírica de este tipo no es una teoría científica si no contiene la formulación de una ley fundamental. Por lo tanto, podemos también decir que se habrá construido una teoría empírica sobre la sección de la realidad $X$ sólo cuando se haya establecido un predicado que denote un concepto cuya extensión sea $X^{28}$.

1.2.1.2. Para construir una teoría empirica desde este punto de vista es necesario, pues, establecer un predicado. Con este fin, por una parte se ha de elegir una expresión lingüística para la denominación del concepto denotado por el predicado y, por otra, se debe determinar exactamente dicho concepto. La solución del primer problema no ofrece ninguna dificultad, ya que tan sólo se trata de asignar un nombre a un concepto. Más problemática, sin embargo, es la segunda tarea. Disponer de un concepto preciso de lo que debe ser una teoría empírica es la condición teórico-científica fundamental en el marco del funcionalismo constructivo ${ }^{29}$. La determinación exacta del concepto denotado por un predicado exige tanto su definición intensional, es decir, la explicación de la estructura lógica de la teoría, como su definición extensional, o sea, la especificación de la sección de la realidad sobre la que se pretende aplicar la teoria ${ }^{30}$. Nuestro principal objetivo a partir de este momento va a ser, por ello, la descripción y explicación metateóricas del más importante componente estructural de la ciencia empírica de la literatura, la teoría empírica de la literatura.

2. Con relación a la definición extensional o a la especificación de la sección de la realidad sobre la que se pretende aplicar la teoría, debemos decir, de acuerdo con lo expuesto en 1.1.1.1, que el objeto de estudio de la ciencia empírica de la literatura es un sistema social de acciones, el sistema social de las acciones literarias o sistema de la LITERATURA ${ }^{31}$. Este sistema, llamado también ámbito de aplicación o

28 Cfr. P. Finke, Konstruktiver Funktionalismus, cit., pp. 144-146, 153, 156 y H. Hauptmeier - S. J. Schmidt, Einführung in die Empirische Literaturwissenschaft, cit., pp. 39-40.

29 Cfr. P. Finke, Konstruktiver Funktionalismus, cit., p. 72.

30 Cfr. Ibid., pp. 144 ss. y H. Hauptmeier - S. J. Schmidt, Einführung in die Empirische Literaturwissenschaft, cit., p. 40.

31 En efecto, para Siegfried J. Schmidt el objeto de estudio más apropiado para una moderna ciencia racional de la literatura en ningún caso está constituido por textos literarios, sino por todas aquellas acciones de naturaleza social que hacen posible la producción, la transmisión o mediación, la recepción y la crítica o transformación de las obras de arte verbal. Con el fin de diferenciar este particular concepto de "literatura" del concepto tradicional se refiere a la LITERATURA y al sistema social de la LITERATURA 
de interpretación previsto / («intendierte Anwendungsbereich») de la teoría empírica de la literatura, constituye su componente empirico y puede ser caracterizado de manera general como el ámbito total de la sección de la realidad que se intenta describir y explicar con la ayuda de esta teoría ${ }^{32}$.

3. Puesto que el sistema de la LITERATURA es muy amplio, es ciertamente difícil que un científico de la literatura se ocupe en su trabajo de la descripción y explicación de la totalidad del mismo. Antes bien se enfrentará al estudio diferenciado de una u otra de las secciones especificas que componen este complejo sistema social. Tomemos como ejemplo para nuestra argumentación la elección de la sección relativa a las acciones literarias de producción. Para esta sección, como para las restantes, la teoria empírica de la literatura dispone de una teoría particular. Se trata en este caso de la teoria de las acciones literarias de producción («Theorie Literarischer Produktionshandlungen") ${ }^{33}$, cuyo componente empirico o ámbito de aplicación o de interpretación previsto / está constituido especificamente por la sección particular del sistema de la LITERATURA correspondiente a aquella serie de acciones en cuyo proceso se elaboran textos lingüísticos que son considerados y tratados por sus autores como textos literarios ${ }^{34}$. Según esta primera aproximación a la explicación de la estructura lógica de la teoría empírica de la literatura y, por lo tanto, a la definición intensional del concepto de su predicado, observamos que dicha teoría empírica está compuesta por una red de teorías empíricas elementales relacionadas entre sí y, a su vez, forma parte de una red de teorías empíricas elementales más generales, como muestra la figura $1^{35}$.

utilizando letras mayúsculas. Cfr. a ese propósito, por ejemplo, S. J. Schmidt, «Empirische Literaturwissenschaft as Perspective», cit;; S. J. Schmidt, Grundriss der Empirischen Literaturwissenschaft. Der gese/lschaftliche Handlungsbereich Literatur, cit.; S. J. Schmidt, "La comunicación literaria", en VV.AA. Pragmática de la comunicación literaria, Madrid, Arco, 1987, pp. 195-212 y H. Hauptmeier - S. J. Schmidt, Einführung in die Empirische Literaturwissenschaft, cit., pp. 14-22.

32 Cfr. P. Finke, Konstruktiver Funktionalismus, cit., pp. 151, 156-157.

33 Cfr. S. J. Schmidt, Grundriss der Empirischen Literaturwissenschaft. Der gesellschaftiche Handlungsbereich Literatur, cit., pp. 199-227 y H. Hauptmeier - S. J. Schmidt, Einführung in die Empirische Literaturwissenschaft, cit., pp. 85-90.

34 Cfr. S. J. Schmidt, Grundiss der Empirischen Literaturwissenschaft. Der gesellschaftiche Handlungsbereich Literatur, cit., pp. 215. Como puede observarse a través de estas explicaciones, para los teóricos de la ciencia empírica de la literatura los conceptos de «literaridad" y de «poeticidad" no dependen ya de las características intrínsecas del texto, sino de dos convenciones sociales, las llamadas convención estética y convención polivalente (cfr. S. J. Schmidt, "Empirische Literaturwissenschaft as Perspective", cit., pp. 562-563; S. J. Schmidt, Grundriss der Empirischen Literaturwissenschaft. Der gesellschaftliche Handlungsbereich Literatur, cit., pp. 86 ss.; S. J. Schmidt, "Fictionality in Literary and Non-Literary Discourse», cit., pp. 542-545; S. J. Schmidt, “ll ruolo delle convenzioni nella comunicazione estetica», en S. J. Schmidt, La comunicazione letteraria, Milano, II Saggiatore, 1983, pp. 57-88; S. J. Schmidt, "The Fiction is that Reality exists", cit., pp. 263-265; S. J. Schmidt, "La comunicación literaria", cit., pp. 202-212 y H. Hauptmeier - S. J. Schmidt, Einführung in die Empirische Literaturwissenschaft, cit., pp. 77-84).

35 En esta figura, donde respetamos las siglas originales, TH representa la teoría de la acción («Theorie der Handlung"), TKH representa la teoria de las acciones comunicativas ("Theorie Kommunikativen Handeins"), TÄKH representa la teoria de las acciones comunicativas estéticas ("Theorie Ästhetischen Kommunikativen Handelns"), TLKH representa la teoria de las acciones comunicativas literarias ("Theorie Literarischen Kommunikativen Handelns"), TLP representa la mencionada teoría de las acciones literarias de producción ( $T$ Theorie Literarischer Produktionshandlungen»), TLV representa la teoria de las acciones literarias de mediación ("Theorie Literarischer Vermittlungshandlungen»), TLR representa la teoría de las acciones 


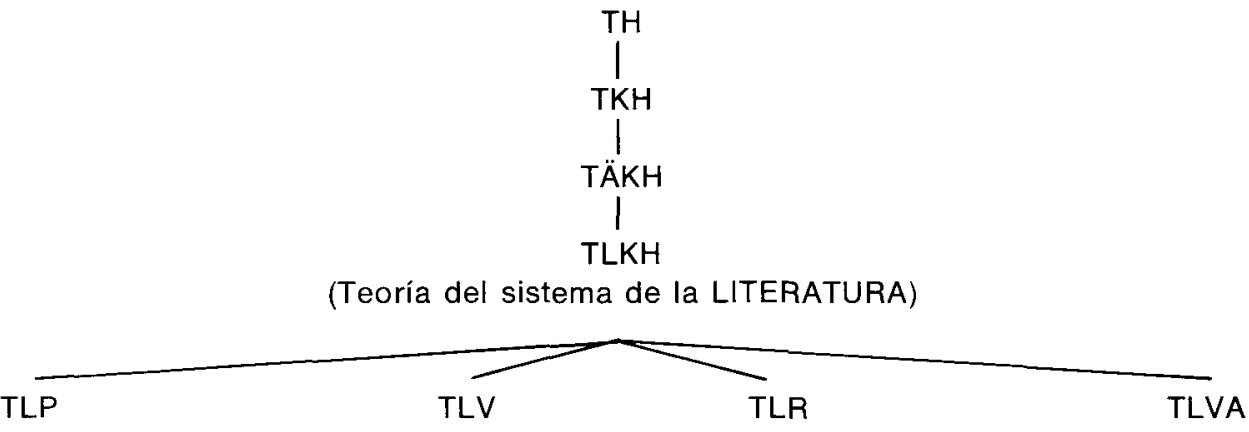

Figura I

3.1. También en una sección particular del sistema de la LITERATURA el científico de la literatura se encuentra, según los postulados del grupo NIKOL, ante un ámbito de aplicación o de interpretación previsto / considerablemente amplio del que debe, en la mayoría de los casos, seleccionar para su estudio determinados elementos específicos. Ahora bien, el científico de la literatura se limita en este momento, por una parte, a considerar esos elementos específicos o estados de cosas como objetos susceptibles de ser descritos y explicados, es decir, interpretados a partir de la teoría en cuestión y, por otra, a describirlos mediante un lenguaje no especializado o mediante la utilización de expresiones científico-literarias tradicionales. En tales descripciones. por ello, van a jugar el principal papel los componentes o conceptos no teóricos (componentes no-t-teóricos) de la teoría, los cuales son no teóricos solamente en relación con la particular teoría de las acciones literarias que se utiliza en ese momento y con su concreto estado de elaboración. Con relación a alguna otra teoría ellos siempre son teóricos ${ }^{36}$, pues, de acuerdo con los presupuestos teórico-cognitivos en los que se basa la ciencia empírica de la literatura y a los que ya nos hemos referido en 1.1.1.1, cualquier descripción empírica, así como cualquier experiencia en el ámbito de la cognición humana, no puede llevarse a cabo más que con la ayuda de teorías ${ }^{37}$. A esos elementos específicos o estados

literarias de recepción ("Literarischer Rezeptionshandlungen») y TLVA representa la teoría de las acciones literarias de transformación ("Theorie Literarischer Verarbeitungshandlungen»). Cfr., en relación con estas teorias elementales de la teoría empírica de la literatura, S. J. Schmidt, Empirische Literaturwissenschaft as Perspective», cit.; S. J. Schmidt, Grundriss der Empirischen Literaturwissenschaft. Der gesel/schaftiche Handlungsbereich Literatur, cit., pp. 11-15 y H. Hauptmeier - S. J. Schmidt, Einführung in die Empirische Literaturwissenschaft, cit., pp. 57-58.

36 Cfr. P. Finke, Konstruktiver Funktionalismus, cit., pp. 151-152.

37 Cfr. S. J. Schmidt, «Empirical Studies in Literature: Introductory Remarks», cit., pp. 319-321; S. J. Schmidt, "Unsere Welt - und das ist alies", cit.; S. J. Schmidt, "Text, Subjekt und Gesellschaft. Aspekte einer konstruktivistischen Semantik», cit. y H. Hauptmeier - S. J. Schmidt, Einführung in die Empirische Literaturwissenschaft, cit., pp. 26-29. 
de cosas descritos mediante los componentes no-t-teóricos de la teoría se les llama modelos potenciales parciales $\left(M_{\mathrm{pp}}\right)$ de la teoría ${ }^{38}$. En el caso de la teoría de las acciones literarias de producción constituyen $M_{\mathrm{pp}}$ aquellas acciones descritas mediante un lenguaje no especializado o mediante la utilización de expresiones científico-literarias tradicionales como acciones orientadas hacia la construcción de textos lingüísticos considerados y tratados por sus autores como textos literarios ${ }^{39}$.

3.2. El siguiente paso que debe dar el científico de la literatura consiste en intentar describir y explicar los $M_{\mathrm{pp}}$ de la teoría con la ayuda de los componentes o conceptos teóricos (componentes $t$-teóricos) de la teoría en cuestión ${ }^{40}$. A aquellas entidades a las que el científico de la literatura se refiere utilizando conjuntamente los componentes no-t-teóricos y los componentes $t$-teóricos se les denomina modelos potenciales $\left(M_{\mathrm{p}}\right)$ de la teoría ${ }^{41}$. Los $M_{\mathrm{p}}$ son exactamente aquellos elementos específicos a los que, a la luz de la teoría en cuestión, se podría preguntar si " $x$ es $F$ ", es decir, si dichas entidades cumplen o no la ley fundamental de la teoria ${ }^{42}$. En el caso de la teoría de las acciones literarias de producción, para pasar de $\operatorname{los} M_{\mathrm{pp}}$ a los $M_{\mathrm{p}}$ deben ser introducidos en aquéllos determinados componentes o conceptos $t$-teóricos como "productor literario", "sistema de presuposiciones literarias del productor literario", "contexto literario de producción», "estrategia literaria de producción», "acción literaria de producción», «resultado de la producción literaria», etc. ${ }^{43}$.

3.2.1. Los $M_{\mathrm{p}}$ de una teoría, desde este punto de vista, pueden ser considerados como teorizaciones de los $M_{\mathrm{pp}}$ de la misma teoría ${ }^{44}$. A su vez, los $M_{\mathrm{pp}}$ de una teoría pueden ser el resultado de la aplicación de una función de reducción $r$ («Restriktionsfunktion») especial, encargada de suprimir los componentes $t$-teóricos de la teoría, sobre los $M_{p}$ de dicha teoria ${ }^{45}$.

3.3. Finalmente se llama modelos $(M)$ de una teoría a aquellos $M_{\mathrm{p}}$ que efectivamente cumplen " $x$ es $F$ ", la ley fundamental de la teoria en cuestión, constituyendo de este modo elementos o estados de cosas pertenecientes a su concreto ámbito de aplicación o de interpretación previsto / descritos y explicados, es decir, interpretados a partir de dicha teoría ${ }^{46}$. Esta ley, en el caso de la teoria de las acciones

38 Cfr. P. Finke, Konstruktiver Funktionalismus, cit., p. 155.

$39 \mathrm{Cfr}$. S. J. Schmidt, Grundriss der Empirischen Literaturwissenschaft. Der gesellschaftliche Handiungsbereich Literatur, cit., p. 215.

40 Cfr. P. Finke, Konstruktiver Funktionalismus, cit., pp. 151-152.

41 Cfr. Ibíd., p. 155.

42 H. Hauptmeier - S. J. Schmidt, Einführung in die Empirische Literaturwissenschaft, cit., pp. 42-43.

43 Cfr. S. J. Schmidt, Grundriss der Empirischen Literaturwissenschaft. Der gesel/schaftliche Handlungsbereich Literatur, cit., p. 215.

44 Cfr. P. Finke, Konstruktiver Funktionalismus, cit., pp. 158-160.

45 Esta función $r$ es, en el fondo, redundante. Por ello Joseph D. Sneed no la introduce explícitamente como componente lógico del núcleo de un elemento teórico en su estudio "Philosophical Problems in the Empirical Science of Science: A Formal Approach" (cit.). Sin embargo, nosotros preferimos tenerla en cuenta porque, como dice Peter Finke y más tarde veremos, puede ser entendida como una función de empirización, frente a su contraria, que constituye una función de teorización (cfr. P. Finke, Konstruktiver Funktionalismus, cit., p. 155).

46 Cfr. Ibid., p. 156. 
literarias de producción, puede ser reproducida lingüisticamente mediante la fórmula « $P$ actúa productivamente en el sistema de la LITERATURA» ${ }^{47}$. De acuerdo con ella, constituirán los $M$ de la teoría de las acciones literarias de producción todas aquellas acciones que puedan ser efectivamente identificadas con la ayuda de los componentes $t$-teóricos de la teoria como acciones literarias de producción, siendo algunos ejemplos de dichos $M$ la producción del Quijote por Miguel de Cervantes, la producción de La Regenta de Leopoldo Alas o la producción de Cántico por parte de Jorge Guillén ${ }^{48}$.

3.4. Los $M_{\mathrm{pp}}$, los $M_{\mathrm{p}}$, los $M$, construidos por el científico de la literatura a partir de la observación de una sección de la realidad determinada, y la función $r$ son componentes estructurales del núcleo formal $K$ ("formale Kern") o componente lógico de las teorías empíricas en general y de la teoría empírica de la literatura en particular ${ }^{49}$. Si una teoría empírica debe servir para describir y explicar aproximativamente una sección determinada de la realidad, que es su ámbito de aplicación o de interpretación previsto $l, y$, con ello, su componente empírico, su componente lógico debe permitir efectuar el mayor número posible de interpretaciones aproximativas de esa sección de la realidad ${ }^{50}$. Ahora bien, para que las interpretaciones aproximativas de / guarden una cierta relación y no sean totalmente independientes entre sí, el componente lógico de la teoría dispone de otro componente estructural esencial. Este componente estructural del núcleo $K$ de una teoríaa lo constituye la restricción $C$ ( «Constraint») o serie $C$ de restricciones de la teoría y opera sobre las entidades teóricas introducidas en la misma, es decir, sobre los $M_{\mathrm{p}}{ }^{51}$. Tales restricciones se pueden entender como condiciones generales de interpretación. En el caso de la teoría de las acciones literarias de producción Siegfried J. Schmidt menciona dos importantes restricciones: la primera y fundamental restricción general (C1) de la teoría está constituida por la condición que obliga a considerar como acciones literarias de producción sólo aquellas acciones de producción cuyos resultados sean valorados por sus actantes como textos literarios; la segunda restricción general (C2) de la teoria, por su parte, viene definida por la condición de que solamente se investiguen aquellas acciones literarias de producción que efectivamente den como resultado un texto ${ }^{52}$. Con ello, el componente lógico de las

47 Cfr. S. J. Schmidt, Grundriss der Empirischen Literaturwissenschaft. Der gesel/schaftiche Handlungsbereich Literatur, cit., p. 216.

48 Cfr. Ibid., p. 215.

49 Cfr. P. Finke, Konstruktiver Funktionalismus, cit., p. 151.

50 Cfr. Ibid., pp. 156-157.

51 Cfr. Ibid., pp. 153, 156. Mientras que Finke habla aqui de "Beschränkungen», Siegfried J. Schmidt (cfr. S. J. Schmidt, Grundriss der Empirischen Literaturwissenschaft. Der gesellschaftliche Handlungsbereich Literatur, cit., p. 215) y Wolfgang Stegmüller (cfr. W. Stegmüller, Probleme und Resultate der Wissenschaftstheorie und Analytischen Philosophie. Band II: Theorie und Erfahrung. Zweiter Halbband: Theorienstrukturen und Theoriendynamik, cit., pp. $111 \mathrm{ss}$.) utilizan el término "Nebenbedingung", siendo, como ya hemos visto, "constraint" el término inglés correspondiente (cfr. J. D. Sneed, "Philosophical Problems in the Empirical Science of Science: A Formal Approach", cit., pp. 122 ss. y W. Balzer - J. D. Sneed, «Verallgemeinerte Netz-Strukturen empirischer Theorien», en W. Balzer - M. Heidelberger (Hrsg.), Zur Logik empirischer Theorien, Berlin/New York, Walter de Gruyter, 1983, p. 121).

52 Cfr. S. J. Schmidt, Grundriss der Empirischen Literaturwissenschaft. Der gesellschaftliche Handlungsbereich Literatur, cit., p. 215. 
teorias empíricas está configurado definitivamente por los componentes estructurales correspondientes a las series de $\operatorname{los} M_{\mathrm{pp}}, M_{\mathrm{p}}, M, C$ y por la función $r$, siendo éstos, por lo tanto, los elementos que definen intensionalmente el concepto denotado por el predicado de dichas teorías, que es su estructura lógica.

3.5. Las teorías empíricas son, pues, construcciones conceptuales muy complejas, que, por lo general, nunca son aplicadas a un único objeto, sino a una serie más o menos amplia de objetos relacionados entre sí, a una sección completa de la realidad. De ahí que la teoría empírica de la literatura, que tiene como finalidad principal la descripción y explicación del sistema de la LITERATURA, esté compuesta, a su vez, como hemos visto, por un instrumental teórico particular para la descripción y explicación de las acciones literarias de producción, por un instrumental teórico particular para la descripción y explicación de las acciones literarias de mediación, por un instrumental teórico particular para la descripción y explicación de las acciones literarias de recepción y por un instrumental teórico particular para la descripción y explicación de las acciones literarias de transformación, instrumentales teóricos particulares que constituyen teorías empíricas elementales de la ciencia de la literatura o elementos teóricos de la teoría empírica de la literatu$\mathrm{ra}^{53}$.

4. Una vez presentados y explicados los dos componentes esenciales de toda teoría empírica, el componente lógico y el componente empírico, conviene profundizar a continuación en la descripción y explicación metateóricas que sobre los conceptos de "teoría empírica" y de "elemento teórico" nos ofrece la visión no proposicional o concepción conceptual de las teorías y, con ella, el funcionalismo constructivo, que sirve de base metateórica para la ciencia empírica de la literatura.

4.1. De acuerdo con lo que llevamos dicho, el presupuesto estructural más general de los elementos teóricos de la teoría empírica de la literatura y, en general, de cualquier teoría empírica construida sobre estos fundamentos concepcionales viene dado por una matriz, la llamada $m+k$-matriz de un elemento teórico ( $" m+k-T h e o r i e e l e m e n t-M a t r i x »)$, que distingue dos series limitadas de componentes $n$ y $t$ con una cuantificación respectiva $m$ y $k$. Dicho presupuesto puede ser representado mediante la siguiente formulación ${ }^{54}$ :

(D1) $X$ es una $m+k-m a t r i z$ de un elemento teórico si y sólo si

(1) $X \in M$

(2) $m$ y $k$ son números enteros positivos $(0<m, 0 \leq k)$

(3) para todo $x \in X$ hay $n^{1}, \ldots, n_{m}, t_{1} \ldots, t_{\mathrm{k}} \in M$, de modo que $x=$ $=\left\langle n_{1}, \ldots, n_{\mathrm{m}}, t_{1}, \ldots, t_{\mathrm{k}}\right\rangle$.

En ella se especifica que $x \in X$ es un $m+k$-tuplo de componentes no-t-teóricos y de componentes $t$-teóricos. (1) expone que $X$ es una serie no vacía. (2) señala una

53 Cfr. P. Finke, Konstruktiver Funktionalismus, cit., pp. 150-158.

54 Cfr. Ibíd., p. 152; W. Balzer - J. D. Sneed, "Verallgemeinerte Netz-Strukturen empirischer Theorien", cit., pp. 117-168, 120 y H. Hauptmeier - S. J. Schmidt, Einführung in die Empirische Literaturwissenschaft, cit., p. 48. 
cantidad determinada de componentes $n$ (componentes no-t-teóricos) y de componentes $t$ (componentes $t$-teóricos). Finalmente (3) explica que $x \in X$ es una construcción ordenada de $n$ y $t$, el llamado $m+k$-tuplo. El número de los componentes $n$, seguún (2), es siempre mayor que $0(0<m)$, lo que hace posible la primera aproximación del científico al estudio de la sección de la realidad que es el ámbito de aplicación o de interpretación previsto / de su teoría, es decir, la configuración de $M_{\mathrm{pp}}$. El número de los componentes $t$, sin embargo, puede tomar el valor $0(0 \leq k)$. Esta matriz, por ello, sirve también de base estructural para aquellos elementos teóricos que no poseen en un momento determinado de su elaboración componente $t$-teórico alguno que permita pasar de $\operatorname{los} M_{\mathrm{pp}}$ de la teoría a determinados $M_{\mathrm{p}}$, hecho que ocurre, por el contrario, cuando $0<k$.

4.2. El núcleo formal $K$ de un elemento teórico, por otra parte, debe considerarse como el aparato conceptual estable y fundamental del mismo. Él constituye el componente lógico del elemento teórico, utilizado por los científicos para llevar a cabo descripciones y explicaciones empíricas del ámbito de aplicación o de interpretación previsto l o componente empírico de dicho elemento teórico. Una explicación formal del concepto de «núcleo de un elemento teórico» es la siguiente ${ }^{55}$ :

(D2) $X$ es el núcleo de un elemento teórico si y sólo si hay $M_{\mathrm{p}}, M_{\mathrm{pp}}, r, M, C, m$ y $k$, de modo que

(1) $X=\left\langle M_{\mathrm{p}}, M_{\mathrm{pp}} r, M, C>\right.$

(2) $M_{\mathrm{p}}$ es una $m+k$-matriz de un elemento teórico

(3) $M_{\mathrm{pp}}=\left\{\left\langle n_{1}, \ldots, n_{\mathrm{m}}\right\rangle /\right.$ hay $t_{1}, \ldots, t_{\mathrm{k}}$, de modo que $\left\langle n_{1}, \ldots n_{\mathrm{m}}, t_{1}, \ldots t_{\mathrm{k}}\right\rangle$ $\left.>\in M_{\mathbf{p}}\right\}$

(4) $r: M_{\mathrm{p}} \rightarrow M_{\mathrm{pp}}$ es una función, de modo que $r\left(n_{1}, \ldots, n_{\mathrm{m}}, t_{1}, \ldots, t_{\mathrm{k}}\right)=$ $=\left\langle n_{1}, \ldots, n_{\mathrm{m}}\right\rangle$

(5) $M \subset M_{\mathrm{p}}$

(6) C es una restricción para $M_{\mathrm{pp}}$.

En esta definición se exponen y explican paso a paso los componentes estructurales del núcleo de un elemento teórico. (1) reúne dichos componentes estructurales, que ya conocemos. (2) incluye en este núcleo la $m+k-m a t r i z$ de un elemento teórico a través de los $M_{\mathrm{p}}$ del mismo, en tanto en cuanto en ellos aparecen unidos en forma de descripciones y explicaciones teóricas los componentes $t$-teóricos y los componentes no-t-teóricos del elemento teórico en cuestión. Complementariamente (3) especifica que existen componentes $t$-teóricos $\left(t_{1}, \ldots, t_{k}\right)$ para ser aplicados a los $M_{\mathrm{pp}}$ del elemento teórico, que permiten pasar determinadas entidades de la serie de $\operatorname{los} M_{\mathrm{pp}}$ a la serie de los $M_{\mathrm{p}}$. (4), por su parte, expresa que la función $r$ se encarga de suprimir de los $M_{\mathrm{p}}$ los componentes $t$-teóricos, dando como resultado a partir de ellos la serie de los $M_{\mathrm{pp}}$. (5) representa la inclusión de la serie de los $M$ en la serie de los $M_{\mathrm{p}}$ del elemento teórico, ya que, como sabemos, la primera serie está constituida por aquellos elementos de la segunda que efectivamente cumplen la ley fundamen-

55 Cfr. P. Finke, Konstruktiver Funktionalismus, cit., p. 154; W. Balzer - J. D. Sneed, «Verallgemeinerte Netz-Strukturen empirischer Theorien», cit., p. 121 y H. Hauptmeier - S. J. Schmidt, Einführung in die Empirische Literaturwissenschaft, cit., pp. 48-49. 
tal de ese elemento teórico. Por último, (6) explica el concepto y el ámbito de aplicación del conjunto de restricciones $C$ del elemento teórico en cuestión.

4.3. En relación con (D2) introducimos seguidamente la definición formal del concepto de "elemento teórico", concepto fundamental para el entendimiento de la estructura lógica de las teorías empíricas ${ }^{56}$ :

(D3) $X$ es un elemento teórico si y sólo si hay $K$ e l, de modo que

(1) $X=\langle K, 1\rangle$

(2) $K=\left\langle M_{\mathrm{p}}, M_{\mathrm{pp}}, r, M, \mathrm{C}>\right.$

(3) $I \subset M_{\mathrm{pp}}$

Según ésta, un elemento teórico cualquiera está compuesto, como se representa en (1), por un núcleo formal $K$ o componente lógico y por un ámbito de aplicación o de interpretación previsto $I$ o componente empírico. (2) especifica la estructura lógica del núcleo ya definida en (D2). (3), finalmente, expone una idea esencial para el tratamiento correcto de todo elemento teórico de una teoria empirica: los elementos que componen el ámbito de aplicación o de interpretación previsto / forman parte de la serie de los $M_{p p}$ del elemento teórico, al ser tanto aquéllos como ésta de carácter no teórico, es decir, de naturaleza empírica, y al constituir el ámbito de aplicación o de interpretación previsto / la concreta sección de la realidad a partir de la cual los científicos elaboran la mencionada serie de los $M_{\mathrm{pp}}$.

4.4. Para la visión no proposicional o concepción conceptual de las teorías un elemento teórico es la mínima unidad constitutiva de una teoría empírica. Por ello, el concepto de «elemento teórico" no basta por sí solo para determinar intensionalmente el concepto de “teoría empírica". Los científicos raras veces trabajan utilizando solamente una teoria más o menos general y, por lo tanto, aplicando una única ley fundamental. Necesitan acudir progresivamente a leyes fundamentales más especiales o menos generales que hagan posible la solución de los problemas cada vez más específicos que se presentan en cualquier sección de la realidad objeto de estudio, lo que conlleva, desde esta perspectiva, la necesidad de trabajar con teorías especiales con diferentes grados de generalidad. Cada sección de la realidad, como la sección constituida por el sistema de la LITERATURA, cumple en verdad una ley fundamental general. Pero cada una de las secciones distinguibles dentro de ella, aparte de estar sujeta a esa ley fundamental general, cumple otras leyes fundamentales especiales o menos generales. En la sección del sistema de la LITERATURA correspondiente a las acciones literarias de producción, ejemplo que ha guiado toda nuestra exposición anterior, la ley fundamental válida es una ley especial con relación a la ley fundamental válida en la totalidad del sistema de la LITERATURA. Todavía tendrá un grado mayor de especialidad o un grado menor de generalidad la ley fundamental aplicable a la sección de las acciones literarias de

56 Cfr. P. Finke, Konstruktiver Funktionalismus, cit., p. 157; W. Balzer - J. D. Sneed, «Veraligemeinerte Netz-Strukturen empirischer Theorien", cit., p. 122 y H. Hauptmeier - S. J. Schmidt, Einführung in die Empirische Literaturwissenschaft, cit., p. 49. 
producción relativas a la producción lírica o a otros tipos concretos de producción literaria. A su vez, la ley fundamental aplicable al sistema de la LITERATURA es una ley fundamental especial con relación a la ley fundamental válida en la sección de la realidad constituida por las acciones comunicativas estéticas, de las que se ocupa, como puede verse en la figura I, un elemento teórico más general que el correspondiente al estudio de las acciones comunicativas literarias, la teoria de las acciones comunicativas estéticas (TÄKH) ${ }^{57}$.

4.4.1. De acuerdo con estas distinciones se llama elemento teórico especializado en el marco metateórico de la visión no proposicional o concepción conceptual de las teorias al elemento teórico cuya ley fundamental es especial con relación a la ley correspondiente a cualquier otro elemento teórico de una teoría empírica determinada. Se denomina, por su parte, elemento teórico básico al elemento teórico que expresa la ley fundamental de una teoría empírica ${ }^{58}$. Las especializaciones tienen, como ha argumentado Peter Finke ${ }^{59}$, el efecto de una lupa, pues que ayudan a aguzar, por decirlo así, la mirada sobre el ámbito de aplicación o de interpretación previsto / del elemento teórico básico. Una especialización o un elemento teórico especializado aplica, por lo tanto, a una serie parcial de / una ley fundamental especial o menos general que restringe la extensión de la serie de los $M$ del elemento teórico básico. La especialización $(\sigma)$ de un elemento teórico básico $T$ en un elemento teórico especializado $T^{\prime}$ es, según todo ello, definible como sigue ${ }^{60}$ :

(D4) Siendo $T^{\prime}$ y $T$ elementos teóricos, $T^{\prime}$ es una especialización de $T\left(T^{\prime} \sigma T\right)$ si y sólo si

(1) $M_{\mathrm{pp}}^{\prime}=M_{\mathrm{pp}}$

(2) $M_{\mathrm{p}}^{\prime}=M_{\mathrm{p}}$

(3) $r^{\prime}=r$

(4) $M^{\prime} \subseteq M$

(5) $C^{\prime} \subseteq C$

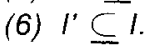

Como se ve en esta definición, los componentes estructurales del elemento teórico especializado correspondientes a las series de $\operatorname{los} M_{\mathrm{pp}}^{\prime}$ y de los $M_{\mathrm{p}}^{\prime}$ y a la relación $r^{\prime}$ se identifican completamente con los componentes respectivos del ámbito del elemento teórico básico, según se muestra en (1), (2) y (3). Ciertamente, en ambos elementos las series de $\operatorname{los} M_{\mathrm{pp}}$ y de los $M_{\mathrm{p}}$ poseen la misma extensión, ya que la ley fundamental especial del elemento teórico especializado no ha sido todavía aplicada sobre la serie de los $M_{\mathrm{p}}$ que sirven de base para las interpretaciones propias del elemento teórico básico, constituyendo todos sus elementos posibilidades de

57 Cfr. S. J. Schmidt, Grundriss der Empirischen Literaturwissenschaft. Der gesellschaftliche Handlungsbereich Literatur, cit., pp. 80-129 y H. Hauptmeier -S. J. Schmidt, Einführung in die Empirische Literaturwissenschatt, cit., pp. 77-84.

58 Cfr. P. Finke, Konstruktiver Funktionalismus, cit., pp. 161-164, 170-172.

59 Cfr. Ibíd., pp. 161-162.

60 Cfr. Ibid., p. 161; W. Balzer - J. D. Sneed, «Verallgemeinerte Netz-Strukturen empirischer Theorien». cit., pp. 125-126 y H. Hauptmeier - S. J. Schmidt, Einführung in die Empirische Literaturwissenschaft, cit., p. 52. 
interpretación generales también para el elemento teórico especializado. Por el contrario, los componentes estructurales del elemento teórico especializado correspondientes a los $M^{\prime}$, al conjunto de restricciones $C^{\prime}$ y al ámbito de aplicación o de interpretación previsto l' se encuentran incluidos dentro de los componentes respectivos pertenecientes al elemento teórico básico, según se muestra en (4), (5) y (6), puesto que en este caso la ley fundamental especial del elemento teórico especializado ha seleccionado del conjunto de $\operatorname{los} M$ una determinada serie de $M^{\prime}$, las restricciones $C^{\prime}$ constituyen condiciones especiales de interpretación presupuestas implícitamente en el conjunto de restricciones $C$ y, por consiguiente, el ámbito de aplicación o de interpretación previsto l' representa una sección parcial del ámbito de aplicación o de interpretación previsto / del elemento teórico básico. La relación interteórica de especialización, como otros tipos de relaciones interteóricas ${ }^{61}$, constituye una operación de extraordinaria importancia en la construcción de teorías empíricas y, como ya hemos podido observar particularmente, en el proceso de construcción de la teoría empírica de la literatura.

4.5. De este modo, la forma lógica de las teorías empíricas es representable como una serie estructurada y ampliable de elementos teóricos o como una red teórica ("Theorienetz») modificable, cuyos nudos son elementos teóricos y sus hilos representan relaciones interteóricas del tipo que acabamos de presentar. Todo elemento teórico de una teoría empírica está, de acuerdo con esta imagen, ligado directa o indirectamente a otros elementos teóricos. De ahí que los científicos que trabajan empíricamente en el marco de cualquier disciplina lo hagan con diferentes elementos teóricos de una red teórica más o menos amplia ${ }^{62}$. La relación existente entre los diferentes elementos teóricos de una red teórica puede ser denominada relación «es-más-general-que» («ist-allgemeiner-als-Beziehung»), puesto que dicha red teórica se presenta como una construcción jerárquicamente ordenada de elementos teóricos que diferencia los elementos teóricos más generales de los más especiales y permite el establecimiento de sucesivos elementos teóricos especializados. Sirve de nuevo como ejemplo paradigmático la representación, en la figura I, de la estructura de la teoria empírica de la literatura, compuesta hasta este momento por ocho elementos teóricos diferenciados y organizados en una red de este tipo de acuerdo con la relación que hemos mencionado.

4.5.1. Para definir intensionalmente el concepto de "teoría empírica», por lo tanto, necesitamos utilizar los siguientes componentes: a) un elemento teórico $<K_{0}$, $I_{0}>$, donde «0» en sus componentes especifica que se trata de un elemento teórico

61 Otras relaciones interteóricas de gran importancia en la construcción de teorias empíricas son las de teorización, complementación y reducción. Para su definición vid. P. Finke, Konstruktiver Funktionalismus, cit., pp. 158-167; W. Balzer - J. D. Sneed, "Verallgemeinerte Netz-Strukturen empirischer Theorien", cit., pp. 123-130 y H. Hauptmeier - S. J. Schmidt, Einführung in die Empirische Literaturwissenschaft, cit. pp. 52-53.

62 Cfr. P. Finke, Konstruktiver Funktionalismus, cit., pp. 167-172. Como dicen Wolfgang Balzer y Joseph D. Sneed, es normal encontrar en las consideraciones introductorias a los trabajos de investigación expresiones como las siguientes: "Teile der Theorie "bauen auf" anderen Teilen auf, sie sind "tieferliegend" als andere, sie "setzen" andere "voraus", einige Teile sind "fundamentaler" als andere, andere sind "Spezialfälle» von andere, einige Teile sind "grundlegend", einige geben "ein detaillierteres" Bild usw." (W. Balzer - J. D. Sneed, "Verallgemeinerte Netz-Strukturen empirischer Theorien», cit., p. 131). 
básico; b) una serie mínima de aplicaciones o de interpretaciones paradigmáticas $\left(I_{\mathrm{p}}\right)$ de $\left\langle K_{0}, I_{0}\right\rangle$ que puedan ser consideradas como investigaciones empiricas ejemplares en procesos concretos de investigación, por lo que el elemento teórico básico debe aparecer justamente representado como $\left.\left\langle K_{0}, l_{\mathrm{p}}\right\rangle{ }^{63} ; \mathrm{c}\right)$ una red teórica paradigmática $N_{\mathrm{p}}$ («paradigmatisches $N$ etz»), cuyos hilos representen relaciones de especialización $\sigma$ y cuyo elemento básico sea precisamente $\left\langle K_{0}, l_{p}\right\rangle$; y d) una relación de expansión $N$ que permita posibles ampliaciones de la red $N_{\mathrm{p}}$ en $N$ («Netz»), ampliaciones simbolizadas mediante el signo " [". De esta forma ${ }^{64}$,

(D5) $X$ es una teoría empirica si y sólo si hay $K_{0}, l_{p}, N_{p}$ y $N$, de modo que

(1) $X=\left\langle K_{0}, I_{p}, N_{p}, N\right\rangle$

(2) $\left\langle K_{0}, I_{p}\right\rangle$ es un elemento teórico de acuerdo con (D3)

(3) $N_{p}$ es una $\sigma$-red teórica con el elemento teórico básico $\left\langle K_{0}, I_{p}\right\rangle$

(4) $N=\left\{\begin{array}{l}\text { a) } N \text { es una } \sigma \text {-red teórica } \\ \text { b) hay } I_{0}, I_{\mathrm{p}}\left[I_{0}\right. \\ \text { de modo que } \\ \\ N=\left\{\left\langle K_{0}, I_{0}\right\rangle\right\} \\ \text { c) } N_{\mathrm{p}}[N .\end{array}\right\}$

Con esta definición queda explicado el concepto de "teoría empírica» y, además, desde un punto de vista diacrónico. Todo sistema teórico que contenga los componentes $K_{0}, I_{p}, N_{p}$ y $N$ puede ser considerado como un sistema teórico empírico y dinámico en tanto que posibilita lógicamente un cambio de estado de una red teórica paradigmática $N_{p}$ en una red teórica ampliada $N$.

5. Ésta es la definición del concepto de «teoria» que sirve de fundamento metateórico para la teoría empírica de la literatura, pues está construida de tal modo que posee la estructura lógica $\left\langle K_{0}, I_{\mathrm{p}}, N_{\mathrm{p}} N\right\rangle$. Una de las ventajas más importantes que ofrece este concepto de "teoría", ventaja reconocida abiertamente por todos los miembros del grupo de investigación NIKOL ${ }^{64}$, es la de hacer posible el tratamiento de los problemas científicos correspondientes a las diferentes secciones que componen el sistema de la LITERATURA tanto teórica como empíricamente, dado que la teoría empírica de la literatura, como cualquier otra teoría construida a partir de la visión no proposicional o concepción conceptual de las teorias, está fundamentada estructuralmente tanto en componentes o conceptos teóricos como en componentes o conceptos no teóricos, teniendo siempre en cuenta, de acuerdo con el presupuesto teórico-cognitivo de la teoricidad del conocimiento humano, que los

63 Cfr. P. Finke, Konstruktiver Funktionalismus, cit, p. 179; W. Balzer - J. D. Sneed, «Verallgemeinerte Netz-Strukturen empirischer Theorien», cit., p. 144 y H. Hauptmeier - S. J. Schmidt, Einführung in die Empirische Literaturwissenschaft, cit., pp. 54-55.

$64 \mathrm{Ctr}$. H. Hauptmeier - S. J. Schmidt, Einführung in die Empirische Literaturwissenschaft, cit., pp. 55-56. 
componentes no teóricos de la teoría empírica de la literatura son en todo caso componentes teóricos de alguna otra teoría. Este concepto de «teoría" es, además, un concepto explicitamente dinámico: las construcciones teóricas de la ciencia empírica de la literatura pueden modificarse, por ejemplo, a través de especializaciones, teorizaciones, complementaciones o reducciones en sus procesos prácticos de investigación sin desvirtuar por ello su organización interna o su identidad, lo que constituye para los teóricos de la ciencia empírica de la literatura una esencial contribución a esta disciplina filológica. Finalmente, por su concreta estructura reticular, para el grupo NIKOL la teoría empírica de la literatura puede facilitar la aproximación a los diferentes problemas literarios que preocupan a los científicos de la literatura de un modo mucho más adecuado que cualquiera de las orientaciones concepcionalmente heterogéneas y teórico-metodológicamente restringidas que caracterizan el presente de la ciencia de la literatura y puede ser fácilmente enseñable y aprendible ${ }^{65}$. No obstante, nosotros hemos de reconocer que la ciencia empírica de la literatura, como consecuencia de su preocupación preferencial por el conjunto de las acciones que definen el complejo sistema de la LITERATURA, no se ha propuesto hasta este momento el estudio intrínseco de la obra de arte verbal, por lo que la teoría empírica de la literatura no posee elemento teórico alguno capaz de dar cuenta de los rasgos lingüísticos especiales que hacen que un texto pueda ser considerado y tratado desde este punto de vista como un texto literario. 